University of Nebraska - Lincoln

DigitalCommons@University of Nebraska - Lincoln

Roger Kirby Publications

Research Papers in Physics and Astronomy

April 1999

\title{
Activation entropy, activation energy, and magnetic viscosity
}

Ralph Skomski

University of Nebraska-Lincoln, rskomski2@unl.edu

Roger D. Kirby

University of Nebraska-Lincoln, rkirby1@unl.edu

David J. Sellmyer

University of Nebraska-Lincoln, dsellmyer@unl.edu

Follow this and additional works at: https://digitalcommons.unl.edu/physics_kirby

Part of the Physics Commons

Skomski, Ralph; Kirby, Roger D.; and Sellmyer, David J., "Activation entropy, activation energy, and magnetic viscosity" (1999). Roger Kirby Publications. 8.

https://digitalcommons.unl.edu/physics_kirby/8

This Article is brought to you for free and open access by the Research Papers in Physics and Astronomy at DigitalCommons@University of Nebraska - Lincoln. It has been accepted for inclusion in Roger Kirby Publications by an authorized administrator of DigitalCommons@University of Nebraska - Lincoln. 


\title{
Activation entropy, activation energy, and magnetic viscosity
}

\author{
R. Skomski, ${ }^{\text {a) }}$ R. D. Kirby, and D. J. Sellmyer \\ Center for Materials Research and Analysis and Behlen Laboratory of Physics, University of Nebraska, \\ Lincoln, Nebraska 68588-0113
}

Starting from an exact quantum-statistical description, the influence of the shape of the energy landscape on the magnetic viscosity is investigated. Magnetic phase-space analysis based on Kramers' escape-rate theory of chemical reaction kinetics theory shows that the activation entropy associated with thermally activated hopping modifies the magnetic viscosity by reducing the attempt-frequency prefactor compared to an earlier prediction by Brown [W. F. Brown, Phys. Rev. 130, 1677 (1963)]. Energetic contributions are analyzed in terms of a model applicable to a range of coherent and noncoherent magnetization processes, and in the long-time limit deviations from the linear logarithmic magnetic-viscosity law are found. (c) 1999 American Institute of Physics.

[S0021-8979(99)74808-5]

\section{INTRODUCTION}

Magnetic viscosity, that is, the time dependence of intrinsic properties such as magnetization and coercivity, is of utmost importance in the areas of hard and semihard magnetism. For example, the stability of the information stored in magnetic and magneto-optic recording media is largely determined by thermally activated magnetization processes (see, e.g., Ref. 1). Magnetic viscosity is most pronounced for small grain sizes, as envisaged in the context of everincreasing magnetic-recording storage densities, and the question arises whether there are contributions going beyond the well-known Arrhenius-type exponential relaxation. A conceptually very simple Arrhenius approach is to consider an ensemble of individual relaxation processes (index $i$ ) described by relaxation times $\tau_{i}=\tau_{o i} \exp \left(E_{a i} / k_{B} T\right)$, where $E_{a i}$ and $\tau_{o i}$ are activation energies and inverse attempt frequencies, respectively, so that the time dependence of the magnetization is an ensemble average. An alternative view is to analyze the sweep-rate dependence of the coercivity in terms of fluctuation fields, ${ }^{1}$ but it can be shown that that approach does not yield essential new physics.

In any case, Fig. 1 shows that the energy barriers $E_{a i}$ are not the only consideration: (a) and (b) have the same activation energy, but the transition (b) is three times as likely as (a). This gives rise to an activation entropy $S_{a i}$ defined by

$$
\tau_{i}=\tau_{o} \exp \frac{E_{a i}-T S_{a i}}{k_{B} T}
$$

Essentially, activation entropy amounts to a renormalized inverse attempt frequency $\tau^{\prime}=\tau_{o} \exp \left(-S_{a i} / k_{B}\right)$, although $S_{i a}$ is in general weakly temperature dependent. In the model illustrated in Fig. 1, the activation entropies are $S_{a i}=0$ (left) and

\footnotetext{
${ }^{a)}$ Electronic mail: rskomski@unlinfo.unl.edu
}

$k_{B} \ln 3$ (right). On the other hand, the question arises of how the shape of the energy landscape affects the energetics of magnetic viscosity.

This article consists of three parts. In Sec. II we summarize the quantum-statistical background of magnetic viscosity and show how the separation of relevant and irrelevant degrees of freedom yields well-defined free-energy landscapes and expressions of the type of Eq. (1). In Sec. III we introduce a magnetic interpretation of Kramers' escape-rate theory of chemical reaction kinetics in terms of an activation-entropy approach, and in Sec. IV we show that the shape of the energy landscape affects not only the entropics but also leads to nontrivial deviations from the famous linear logarithmic magnetic-viscosity law.

\section{QUANTUM-STATISTICAL BACKGROUND}

The evolution of a quantum-mechanical system is described by the time-dependent Schrödinger equation $i \hbar \partial|\Psi\rangle / \partial t=\mathcal{H}|\Psi\rangle$ or, alternatively, by the Liouville-von Neumann equation

$$
i \hbar d \hat{\rho} / d t=\mathcal{H} \hat{\rho}-\hat{\rho} \mathcal{H},
$$
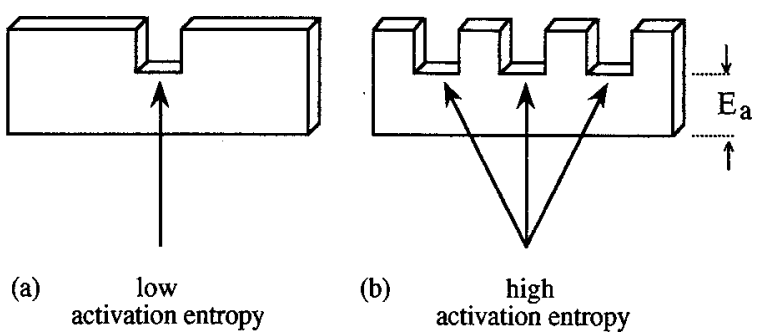

FIG. 1. Activation energy $E_{a}(\underline{s})$, activation entropy, and the number of phase-space paths in a two-dimensional phase space. 
where $\hat{\rho}(t)=|\Psi(t)\rangle\langle\Psi(t)|$ is the density operator. Equation (2) can be used to predict the evolution of any physical system, but from the time dependence of the entropy

$$
d S / d t=-\left(k_{B} / i \hbar\right) \operatorname{Tr}(\ln \hat{\rho} \mathcal{H} \hat{\rho}-\ln \hat{\rho} \hat{\rho} \mathcal{H})=0,
$$

we see that this method is not feasible in practice. The reason is the deterministic character of the many-body Schrödinger and Liouville-von Neumann equations, whereas irreversibility is associated with the transition from the complete Hamiltonian to a "coarse-grained" Hamiltonian describing the relevant magnetic degrees of freedom, such as the position of a domain wall, whereas the irrelevant degrees of freedom, such as lattice vibrations and magnons, act as a heat bath.

Irrelevant degrees of freedom are eliminated by introducing projection operators so that $\hat{\rho}_{\text {rel }}=\mathbf{P} \hat{\rho}$ and $\hat{\rho}^{\prime}=(\mathbf{1}$ $-\mathbf{P}) \hat{\rho}=\mathbf{Q} \hat{\rho}$ are the relevant and irrelevant components of the density operator, respectively. ${ }^{2,3}$ Writing Eq. (2) as $i \hbar \partial \hat{\rho} / \partial t=\mathbf{L} \hat{\rho}$, where $\mathbf{L}$ is the Liouville superoperator, yields the coupled equations $i \hbar \partial \hat{\rho}_{\text {rel }} / \partial t=\mathbf{P} \mathbf{L} \hat{\rho}_{\text {rel }}+\mathbf{P L} \hat{\rho}^{\prime}$ and $i \hbar \partial \hat{\rho}^{\prime} / \partial t=\mathbf{Q L} \hat{\rho}_{\text {rel }}+\mathbf{Q L} \hat{\rho}^{\prime}$. The second equation describes the heat bath. Solving it and substituting into the first equation yields a closed equation for $\hat{\rho}_{\text {rel }}(t)$

$$
\begin{aligned}
i \hbar \frac{\partial \hat{\rho}_{\text {rel }}}{\partial t}= & \mathbf{P L} \hat{\rho}_{\text {rel }}-\frac{i}{\hbar} \int_{0}^{t} \mathbf{P L} \exp \left(-\frac{i\left(t-t^{\prime}\right)}{\hbar} \mathbf{Q L}\right) \\
& \times \mathbf{Q} \mathbf{L} \hat{\rho}_{\text {rel }}\left(t-t^{\prime}\right) d t^{\prime}+\mathbf{P L} \exp \left(-\frac{i t}{\hbar} \mathbf{Q} \mathbf{L}\right) \hat{\rho}^{\prime}(0) .
\end{aligned}
$$

In this exact but formal master equation, the term PL $\hat{\rho}_{\text {rel }}$ reflects the deterministic quantum-mechanical motion of the relevant degrees of freedom, whereas the second and third terms on the right-hand side of Eq. (4) describe relaxation processes and random thermal forces associated with the heat bath, respectively.

Equation (4) contains difficult-to-handle operator expressions, but it provides a sound basis for the derivation of approximations. For example, the Landau-Lifshitz equation includes the precession of the magnetization and the viscous rotation of $\mathbf{M}$ towards an effective field $\mathbf{H}_{\mathrm{eff}}=$ $-\delta E(\mathbf{M}) / \delta\left(\mu_{o} \mathbf{M}\right)$, but it is unable to explain thermally activated transitions (jumps) over energy barriers. A more appropriate approach to magnetic viscosity is the Langevin equation

$$
\frac{\partial s}{\partial t}=-\frac{\Gamma_{o}}{k_{B} T} \frac{\partial E}{\partial \underline{s}}+\sqrt{2 \Gamma_{o}} \xi(t),
$$

where $\underline{s}$ is a magnetic phase-space vector, $\Gamma_{o}=1 / \tau_{o}$, and $\xi(t)$ is a delta-correlated random force. Strictly speaking, the number of components of $\underline{s}$ is infinite, because for $|\mathbf{M}(\mathbf{r})|$ $=M_{s}(\mathbf{r})$ the magnetization $\mathbf{M}(\mathbf{r})$ has two degrees of freedom $\theta$ and $\phi$ per volume element $d \mathbf{r}$, but it is common to consider suitably projected low-dimensional phase spaces. For example, considering the position of a domain wall establishes a one-dimensional phase space. The probability distribution $P(\underline{s}, t)$ obeys the magnetic Fokker-Planck equation

$$
\tau_{o} \frac{\partial P}{\partial t}=\frac{1}{k_{B} T} \frac{\partial}{\partial \underline{s}}\left(P \frac{\partial E}{\partial \underline{s}}\right)+\frac{\partial^{2} P}{\partial \underline{s}^{2}},
$$

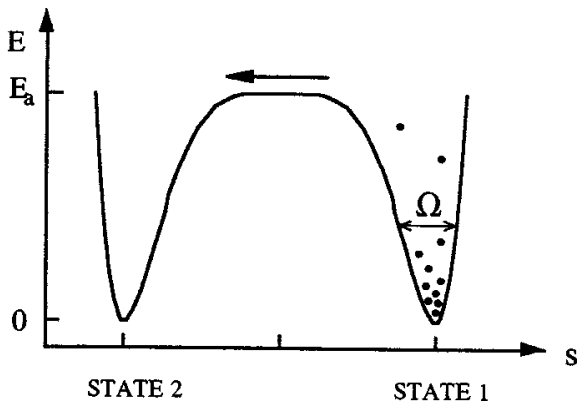

FIG. 2. Model potential illustrating the process of magnetic viscosity; the filled arrow shows the net probability flux. This model describes, for example, a Barkhausen jump of a plane domain wall from the wall position $s_{1}$ to the wall position $s_{2}$.

which can be interpreted as a generalized diffusion equation (see, e.g., Ref. 4, and references therein), and both Eqs. (5) and (6) can be derived from a phenomenological master or rate equation

$$
\frac{\partial P(\underline{s})}{\partial t}=\int\left[W\left(\underline{s}, \underline{s}^{\prime}\right) P\left(\underline{s}^{\prime}\right)-W\left(\underline{s}^{\prime}, \underline{s}\right) P(\underline{s})\right] d \underline{s}^{\prime},
$$

where the $W\left(\underline{s}, \underline{s}^{\prime}\right)=W\left(\underline{s}^{\prime} \rightarrow \underline{s}\right)$ are appropriately chosen transition rates. In equilibrium, Eqs. (5), (6), and (7) all reproduce the Boltzmann distribution $P(\underline{s})=(1 / Z) \exp$ $\left(-E(\underline{s}) / k_{B} T\right)$, whereas in nonequilibrium they yield expressions of the type of Eq. (1). Note that fast heat-bath motions determine, for example, the spontaneous magnetization $M_{s}(T)$, so that $E(\underline{s})$ is a temperature-dependent quasiequilibrium magnetic free energy.

\section{ACTIVATION ENTROPY AND KRAMER'S ESCAPE- RATE THEORY}

A simplified master equation, $\partial P_{1} / \partial t=W_{12} P_{2}-W_{21} P_{1}$ and $\partial P_{2} / \partial t=-W_{12} P_{2}+W_{21} P_{1}$, describing net transitions between two global or local energy minima, is illustrated in Fig. 2. Kramers' escape-rate theory, ${ }^{5}$ originally used to describe chemical reaction kinetics, uses Eq. (6) to show that the transition rates $W_{12}$ and $W_{21}$ are proportional to exp $\left(-E_{a} / k_{B} T\right)$ but also contain a prefactor which depends on the curvature of the energy extrema. In our approach, we can define entropy as $S=k_{B} \ln \Omega$, where $\Omega(E)$ is the available phase-space volume (Fig. 2). A small phase-space volume at the bottom of the metastable energy minimum $\left(E \ll E_{a}\right)$ is entropically favorable, because $S_{a}=k_{B}\left[\ln \Omega\left(E_{a}\right)-\ln \Omega(E)\right]$ is large. Quadratic potentials $E \approx k\left(s-s_{o}\right)^{2}$, where $\Omega \sim 1 / \sqrt{k}$, yield, therefore, prefactors proportional to $\sqrt{k}$. This interest-

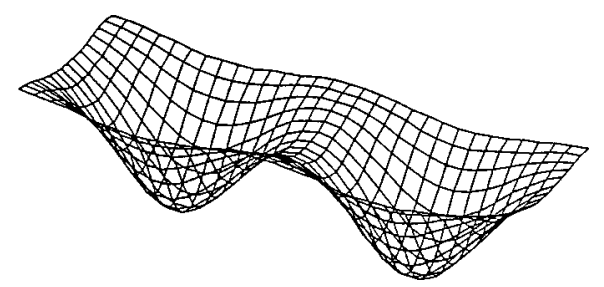

FIG. 3. Two-dimensional energy landscape. The activation entropy is determined by the curvatures at the saddle-point and at the bottom of the initial minimum (left). 
ing result is equivalent to Kramers' approach, where a direct probability-flux integration was used to calculate the reaction rate. Note that $1 / k$ is proportional to the reversible susceptibility $\chi_{\mathrm{rev}}$, so that magnetic viscosity increases with decreasing $\chi_{\text {rev }}$.

In general, magnetization processes are multidimensional phenomena, characterized by two or more relevant degrees of freedom (Fig. 3). For a given energy landscape $E(\underline{s})$, which can be determined by several methods, ${ }^{6,7}$ the rate prefactor can be obtained by solving Eq. (6), as known from reaction-rate theory. ${ }^{8}$ The important result is

$$
\Gamma_{o}=c_{o} \frac{\Pi_{i} \sqrt{k_{i}} \sqrt{\left|k_{a}\right|}}{\Pi_{j} \sqrt{k_{j}^{\prime}}} .
$$

Here, the $k_{i}(i=1, \ldots, N)$ are the curvature (inversesusceptibility) eigenvalues of the metastable energy minimum, the $k_{j}^{\prime}(j=1, \ldots, N-1)$ are the positive curvature eigenvalues of the saddle point, and $k_{a}$ is the negative saddle-point eigenvalue responsible for the bottleneck (Fig. 3).

A notable deviation from Eq. (8) is the magnetic reversal in ideal Stoner-Wohlfarth particles, which involves a degenerate saddle point at $\theta=\pi / 2$. Due to the high symmetry of the problem, Eq. (6) can be solved explicitly, and one obtains a prefactor proportional to $1 / \sqrt{T}{ }^{9}$ However, in reality this symmetry is broken, for example, by magnetostatic interactions with neighboring grains, and $\Gamma_{o}$ is reduced with respect to Brown's prediction. For grains of radius $10 \mathrm{~nm}$, this symmetry breaking reduces the the room-temperature magnetic viscosity by a factor of order 50 .

\section{THE LOGARITHMIC LAW}

As a rule, slow relaxation processes such as the famous logarithmic law $\langle M(t)\rangle=\left\langle M\left(t_{o}\right)\right\rangle-S_{\text {visc }} \ln \left(t / t_{o}\right)$ are based on energy-barrier distributions, ${ }^{10}$ although there is an equivalent logarithmic dependence of the coercivity on the sweep-rate $d H / d t,{ }^{11}$ Trivially, the logarithmic law is unphysical not only in the short-time limit, where spin precession is important, but also for extremely long times, where $\langle M\rangle=-M_{s}$ rather than $\langle M\rangle=-\infty$. The main reason for the trivial longtime deviations is the large but finite width of the energybarrier distribution. ${ }^{10}$ A more adequate expression is ${ }^{12}$

$$
M(t)=2 M_{s}\left(t / \tau_{o}\right)^{-k_{B} T / E_{o}}-M_{s},
$$

but since $\left(x^{\epsilon}-1\right)=\epsilon \ln x$ for small exponents, the deviations from the logarithmic law are of minor interest in the present context.

From Kramers' theory, that is, neglecting nanosecond corrections, it follows that the individual relaxation processes obey $M_{i}(t)=-M_{s}+2 M_{s} \exp \left(-t / \tau_{i}\right)$. This yields the average magnetization

$\langle M(t)\rangle=-M_{s}+2 M_{s} \int_{-\infty}^{\infty} P(E) e^{-\Gamma_{o} t \exp \left(-E / k_{B} T\right)} d E$,

where $P(E)$ is the energy-barrier distribution. Since $E$ $\gg k_{B} T$ for most activation energies we can restrict ourselves to the vicinity of the individual switching fields (coercivities) $H_{s}$. Consider, for simplicity, the energy expression

$$
E=\mu_{0} M_{S} V_{0} H_{C}^{1-m}\left(H_{s}-H\right)^{m},
$$

where $V_{o}$ is a physical, Barkhausen-type volume and $H_{c}$ is needed for dimensional reasons. Since there is equilibrium on a local scale (Sec. II), the parameters in Eq. (11) exhibit a secondary temperature dependence. Equation (11) describes various coherent and incoherent magnetization processes (compare, e.g., Ref. 13): it applies to energy barriers of the types $H-H_{o}$ and $1 / H-1 / H_{o}(m=1)$, but it can also be used to describe strong domain-wall pinning $(m=3 / 2)$, oriented fine particles $(m=2)$, and misaligned fine particles $(m$ $=3 / 2$ ). In Eq. (10), the energy-barrier distribution $P(E)$ arises from the randomness of $H_{s}$, that is, from the switching-field distribution of order $M_{s} / \chi_{\text {irr }}$, whereas the prefactor is assumed to be constant. After some calculation we obtain from Eqs. (10) and (11)

$$
\langle M(t)\rangle=\left\langle M\left(\tau_{o}\right)\right\rangle-\left(\frac{k_{B} T H_{c}^{m-1} \ln \left(t / \tau_{o}\right)}{\mu_{o} M_{s} V_{o}}\right)^{1 / m} \chi_{\mathrm{irr}} .
$$

For $m>1$, this equation differs from the popular "logarithmic-law" time-dependence $\ln \left(t / t_{o}\right)$, where $t_{o}$ is a reference time. For small ratios $t / t_{o}$ it is possible to linearize Eq. (12) with respect to $\ln \left(t / t_{o}\right)$, which resurrects the familiar linear dependence on $\ln \left(t / t_{o}\right)$ and involves the well-known expression $\ln \left(t_{o} / \tau_{o}\right) \approx 25$. However, the validity of this linearization implies that $\ln \left(t / t_{o}\right) \ll 25$, rather than, e.g., $k_{B} T \ll E$. This criterion is fairly well satisfied for laboratory-scale measurements with time scales ranging from a few seconds to a few hours, so that $\ln \left(t / t_{o}\right) \approx 8$, but it is violated when longtime data storage is considered, where $\ln \left(t / t_{o}\right) \approx 20$. In this case, there are pronounced deviations from the linear logarithmic law which depend on details of the energy landscape.

\section{v. CONCLUSIONS}

We have investigated how the shape of the energy landscape affects magnetic viscosity. For a given energy-barrier height, there are both entropic and energetic corrections to the magnetic viscosity. The concept of activation entropy is used to show that the curvature of the energy landscape, and reduces the prefactor $\Gamma_{o}$ compared to Brown's prediction, whereas the energetic contribution yields deviations from the simple logarithmic law in the limit of long-time data storage.

\section{ACKNOWLEDGMENT}

This work was supported by NSF Grant No. DMR9623992, AFOSR-F Grant No. 496209810098, ARO/ DARPA Grant No. DAAG55-98-1-0268, and CMRA.

${ }^{1}$ D. J. Sellmyer, M. Yu, R. A. Thomas, Y. Liu, and R. D. Kirby, Phys. Low-Dimens. Semicond. Struct. 1-2, 155 (1998).

${ }^{2}$ R. Zwanzig, Phys. Rev. 124, 983 (1961).

${ }^{3}$ H. Mori, Prog. Theor. Phys. 33, 423 (1965).

${ }^{4}$ R. Skomski, J. Appl. Phys. 83, 6503 (1998).

${ }^{5}$ H. A. Kramers, Physica (Amsterdam) 7, 284 (1940).

${ }^{6}$ W.-J. Chen, Sh.-F. Zhang, and H. N. Bertram, J. Appl. Phys. 71, 5579 (1992)

${ }^{7}$ D. V. Berkov, J. Appl. Phys. 83, 7390 (1998).

${ }^{8}$ P. Hänggi, P. Talkner, and M. Borkovec, Rev. Mod. Phys. 62, 251 (1990).

${ }^{9}$ W. F. Brown, Phys. Rev. 130, 1677 (1963).

${ }^{10}$ R. Becker and W. Döring, Ferromagnetismus (Springer, Berlin, 1939).

${ }^{11}$ L. Néel, J. Phys. Radium 12, 339 (1951).

${ }^{12}$ R. Skomski and V. Christoph, Phys. Status Solidi B 156, K149 (1989).

${ }^{13}$ P. Gaunt, J. Appl. Phys. 59, 4129 (1986). 